\title{
Similarity and Kirillov-Schilling-Shimozono Bijection
}

\section{Masato Okado}

\begin{tabular}{|c|l|}
\hline Citation & Algebras and Representation Theory, 19(4); 975-989 \\
\hline Issue Date & $2016-08$ \\
\hline Type & Journal Article \\
\hline Textversion & Author \\
\hline Relation & $\begin{array}{l}\text { This is a post-peer-review, pre-copyedit version of an article published in Algebras and } \\
\text { Representation Theory. The final authenticated version is available online at: } \\
\text { https://doi.org/10.1007/s10468-016-9607-6 }\end{array}$ \\
\hline DOI & $10.1007 / \mathrm{s} 10468-016-9607-6$ \\
\hline
\end{tabular}

\author{
Self-Archiving by Author(s) \\ Placed on: Osaka City University
}




\title{
SIMILARITY AND KIRILLOV-SCHILLING-SHIMOZONO BIJECTION
}

\author{
MASATO OKADO
}

\begin{abstract}
The behavior of the Kirillov-Schilling-Shimozono bijection is examined under the similarity map on Kirillov-Reshetikhin crystals. It enables us to define this bijection over $\mathbb{Q}$. Conjectures on the extension to $\mathbb{R}$ are also presented.
\end{abstract}

\section{INTRODUCTION}

The Kerov-Kirillov-Reshetikhin bijection [9, 10], or Kirillov-Schilling-Shimozono bijection in more general setting [11], describes a one-to-one correspondence between highest weight elements of a multiple tensor product of Kirillov-Reshetikhin crystals of type A $[6,21]$ and certain combinatorial objects called rigged configurations. Let $B$ be a tensor product of Kirillov-Reshetikhin crystals and $\mathcal{P}(B)$ be the set of highest weight elements of $B$. Then there corresponds a datum $L(B)$, the set $\mathcal{R C}(L(B))$ of rigged configurations associated to $L(B)$, and there exists a bijection

$$
\Phi: \mathcal{P}(B) \longrightarrow \mathcal{R C}(L(B)) \text {. }
$$

The Kirillov-Schilling-Shimozono bijection, KSS bijection for short, has various applications. There exist notions of weight and energy or charge statistic on both sets, and $\Phi$ preserves them. Hence, taking generating functions with a fixed weight give rise to an identity known as $X=M[3,2]$. Using the Kyoto path model [5] $M$ in a suitable limit gives an explicit form of a branching function of the highest weight $\widehat{\mathfrak{s l}}_{n}$-module with respect to the underlying simple Lie algebra $\mathfrak{s l}_{n}$. Another significant application is found in the box-ball system [23], where the bijection $\Phi$ linearize this nonlinear ultra-discrete dynamical system [12].

In [17] we reported that Kirillov-Reshetikhin (KR) crystals have the similarity property. Let $B^{r, s}$ stand for a KR crystal where $r$ is an index of the Dynkin diagram and $s$ a positive integer. The similarity map $S_{m}\left(m \in \mathbb{Z}_{>0}\right)$ is an injective map $S_{m}: B^{r, s} \rightarrow B^{r, m s}$ satisfying some properties on crystal operators. For type A, representing elements of $B^{r, s}$ by semistandard tableaux of $r \times s$ rectangular shape, the image of $S_{m}$ is obtained by enlarging it horizontally $m$ times. Then it is a natural question to ask how the composition map $\Phi \circ S_{m}$ is described. The answer is quite simple and natural. A rigged configuration $(\nu, J)$ is composed of a sequence of partitions $\nu$ and a set of nonnegative integers $J$. The similarity map $S_{m}$ on $\mathcal{R C}(L(B))$ amounts to multiplying by $m$ each part of all partitions in $\nu$ and each integer in $J$ (Theorem 3.3).

The above theorem enables us to consider the KSS bijection over $\mathbb{Q}$. Namely, we represent an element of $B^{r, s}$ and of $\mathcal{P}(B)$ as an integer point of a certain polytope in the Euclidian space. We then consider any rational points of the polytope, apply $\Phi \circ S_{m}$ ( $m$ is chosen so that the image of $S_{m}$ is an integer point), and shrink by 
$m$ on the rigged configuration side. We can show the map so constructed is welldefined and extends the bijection from integer points to rational ones (Proposition 4.1). Furthermore, one should be able to consider $\Phi$ over $\mathbb{R}$. However, the proof of well-definedness seems nontrivial and we leave it to future problem. We end with conjectures on this extension to $\mathbb{R}$ and a connection to the tropical periodic Toda lattice by $[22]$.

\section{REVIEWS ON KR CRYSTALS, PATHS AND RIGGED CONFIGURATIONS}

2.1. KR crystal. A Kirillov-Reshetikhin crystal, KR crystal for short, is a crystal basis of certain finite-dimensional quantum affine algebra module called KirillovReshetikhin module. If the corresponding affine algebra is of nonexceptional type, its existence was shown in [18]. A KR crystal is denoted by $B^{r, s}$, since it is parametrized by $(r, s) \in I \backslash\{0\} \times \mathbb{Z}_{>0}$ where $I$ is the index set of the Dynkin diagram of the affine algebra and 0 is the index as prescribed in [4]. In this paper we denote Kashiwara operators by $e_{i}, f_{i}$.

In [17] we showed the following property of KR crystals of nonexceptional type.

Theorem 2.1. For $m \in \mathbb{Z}_{>0}$ there exists a unique injective map

$$
S_{m}: B^{r, s} \longrightarrow B^{r, m s}
$$

satisfying

$$
S_{m}\left(e_{i} b\right)=e_{i}^{m} S_{m}(b), S_{m}\left(f_{i} b\right)=f_{i}^{m} S_{m}(b)
$$

for $i \in I$ and $b \in B^{r, s}$. Here $S_{m}(0)$ is understood to be 0 .

In what follows we consider the case of $A_{n}^{(1)}$. Set $I_{0}=I \backslash\{0\}$. Let $\alpha_{i}(i \in I)$ be simple roots and $\varpi_{i}\left(i \in I_{0}\right)$ level 0 fundamental weights. We describe the KR crystal $B^{r, s}$ of $A_{n}^{(1)}$. As a set $B^{r, s}$ is given by semistandard tableaux of shape $\left(s^{r}\right)$ with letters from $\{1,2, \ldots, n+1\}$. The action of Kashiwara operators $e_{i}, f_{i}$ for $i \in I_{0}$ is described in [7] by reading letters of the tableau in a certain manner. For $e_{0}, f_{0}$ it is defined through the so called promotion operator pr [21] by

$$
e_{0}=\mathrm{pr}^{-1} \circ e_{1} \circ \mathrm{pr}, \quad f_{0}=\mathrm{pr}^{-1} \circ f_{1} \circ \mathrm{pr} \text {. }
$$

See also $[16, \S 2.2]$ on these descriptions. For an element $b$ of $B^{r, s} S_{m}(b)$ is described as follows. For each row a node with letter $a$ is replaced with $m$ nodes with the same letter $a$.

2.2. Path. Let $B_{1}, B_{2}$ be crystals. The tensor product of crystals $B_{2} \otimes B_{1}$ is defined with its crystal structure given by

$$
\begin{aligned}
& e_{i}\left(b_{2} \otimes b_{1}\right)= \begin{cases}b_{2} \otimes e_{i} b_{1} & \text { if } \varepsilon_{i}\left(b_{2}\right) \leq \varphi_{i}\left(b_{1}\right) \\
e_{i} b_{2} \otimes b_{1} & \text { if } \varepsilon_{i}\left(b_{2}\right)>\varphi_{i}\left(b_{1}\right),\end{cases} \\
& f_{i}\left(b_{2} \otimes b_{1}\right)= \begin{cases}b_{2} \otimes f_{i} b_{1} & \text { if } \varepsilon_{i}\left(b_{2}\right)<\varphi_{i}\left(b_{1}\right) \\
f_{i} b_{2} \otimes b_{1} & \text { if } \varepsilon_{i}\left(b_{2}\right) \geq \varphi_{i}\left(b_{1}\right) .\end{cases}
\end{aligned}
$$

Here $0 \otimes b$ and $b \otimes 0$ are understood by 0 and

$$
\varepsilon_{i}(b)=\max \left\{m \geq 0 \mid e^{m} b \neq 0\right\}, \quad \varphi_{i}(b)=\max \left\{m \geq 0 \mid f^{m} b \neq 0\right\} .
$$

From (2.1), (2.2) we have

$$
\begin{aligned}
\varepsilon_{i}\left(b_{2} \otimes b_{1}\right) & =\max \left(\varepsilon_{i}\left(b_{1}\right), \varepsilon_{i}\left(b_{1}\right)+\varepsilon_{i}\left(b_{2}\right)-\varphi_{i}\left(b_{1}\right)\right), \\
\varphi_{i}\left(b_{2} \otimes b_{1}\right) & =\max \left(\varphi_{i}\left(b_{2}\right), \varphi_{i}\left(b_{1}\right)+\varphi_{i}\left(b_{2}\right)-\varepsilon_{i}\left(b_{2}\right)\right) .
\end{aligned}
$$


As seen from above we use the anti-Kashiwara convention for the tensor product of crystals.

It is known [5] that for $\mathrm{KR}$ crystals $B^{r, s}, B^{r^{\prime}, s^{\prime}}$ there exists an isomorphism of crystals

$$
R: B^{r, s} \otimes B^{r^{\prime}, s^{\prime}} \longrightarrow B^{r^{\prime}, s^{\prime}} \otimes B^{r, s},
$$

called combinatorial $R$-matrix. $R$ commutes with $e_{i}, f_{i}(i \in I)$. The image of $R$ is given as follows [21]. Suppose $R\left(b \otimes b^{\prime}\right)=\tilde{b}^{\prime} \otimes \tilde{b}$. Then $b \leftarrow \operatorname{row}\left(b^{\prime}\right)=\tilde{b}^{\prime} \leftarrow \operatorname{row}(\tilde{b})$, where $\operatorname{row}(b)$ is the row word of $b$, word reading from left to right, bottom to top, and $T \leftarrow w d$ stands for the tableau given by row inserting the word $w d$ into $T$. This condition uniquely determines $\tilde{b}^{\prime}$ and $\tilde{b}$ from $b \otimes b^{\prime}$.

Let $B=B_{k} \otimes \cdots \otimes B_{1}$ be a multiple tensor product of KR crystals. An element of $b$ of $B$ is called a (highest-weight) path if $e_{i} b=0$ for any $i \in I_{0}$. The set of paths in $B$ is denoted by $\mathcal{P}(B)$.

\section{Example 2.1.}

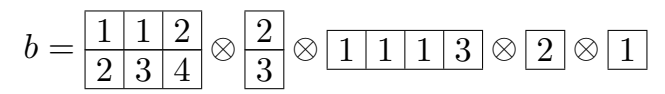

is an element of $\mathcal{P}\left(B^{2,3} \otimes B^{2,1} \otimes B^{1,4} \otimes\left(B^{1,1}\right)^{2}\right)$ of weight $6 \varpi_{1}+4 \varpi_{2}-\left(4 \alpha_{1}+4 \alpha_{2}+\alpha_{3}\right)$.

2.3. Rigged configuration. We concentrate on rigged configurations of type $A_{n}^{(1)}$. Let $\left(C_{a b}\right)_{a, b \in I_{0}}$ be the Cartan matrix of $A_{n}$, that is, $C_{a b}=2 \delta_{a, b}-\delta_{a, b+1}-\delta_{a, b-1}$. Consider a matrix $L=\left(L_{i}^{(a)}\right)_{a \in I_{0}, i \in \mathbb{Z}_{>0}}$ of nonnegative integers, almost all zero. $L$ is called a multiplicity array. Let $\nu=\left(m_{i}^{(a)}\right)$ be another such matrix. Say that $\nu$ is an admissible configuration if it satisfies

$$
p_{i}^{(a)} \geq 0 \quad \text { for any } a \in I_{0} \text { and } i \in \mathbb{Z}_{>0},
$$

where

$$
p_{i}^{(a)}=\sum_{j \in \mathbb{Z}_{>0}}\left(L_{j}^{(a)} \min (i, j)-\sum_{b \in I_{0}} C_{a b} \min (i, j) m_{j}^{(b)}\right) .
$$

$p_{i}^{(a)}$ is called a vacancy number.

Let $\nu=\left(m_{i}^{(a)}\right)_{a \in I_{0}, i \in \mathbb{Z}_{>0}}$ be an admissible configuration. We identify $\nu$ with a sequence of partitions $\left(\nu^{(a)}\right)_{a \in I_{0}}$ such that

$$
\nu^{(a)}=\left(1^{m_{1}^{(a)}} 2^{m_{2}^{(a)}} \cdots\right) .
$$

One can also identify the partition $\nu^{(a)}$ with a Young diagram whose number of rows of length $i$ is $m_{i}^{(a)}$. A rigging $J$ on $\nu$ is to associate, with each part of the Young diagram $\nu^{(a)}$ of the same width $i$, a partition $\left(J_{1}^{(a, i)} \geq J_{2}^{(a, i)} \geq \ldots \geq J_{m_{i}^{(a)}}^{(a, i)}\right)$ of length at most $m_{i}^{(a)}$ such that $p_{i}^{(a)} \geq J_{1}^{(a, i)}$. A pair $(\nu, J)$ of an admissible configuration $\nu$ and a rigging $J$ on $\nu$ is called a rigged configuration.

For a partition $\mu$ and $i \in \mathbb{Z}_{>0}$, define

$$
Q_{i}(\mu)=\sum_{j} \min \left(\mu_{j}, i\right)
$$

the area of $\mu$ in the first $i$ columns. Then the vacancy number (2.6) is rewritten as

$$
p_{i}^{(a)}=Q_{i}\left(L^{(a)}\right)+Q_{i}\left(\nu^{(a-1)}\right)+Q_{i}\left(\nu^{(a+1)}\right)-2 Q_{i}\left(\nu^{(a)}\right) .
$$


Here $L^{(a)}$ is a partition $\left(1^{L_{1}^{(a)}} 2^{L_{2}^{(a)}} \cdots\right)$, and both $\nu^{(0)}$ and $\nu^{(n+1)}$ should be considered as an empty partition. The set of rigged configurations with multiplicity array $L$ is denoted by $\mathcal{R C}(L)$. We define a weight of the rigged configuration $(\nu, J)$ by

$$
\lambda=\sum_{a \in I_{0}}\left(Q_{\infty}\left(L^{(a)}\right) \varpi_{a}-Q_{\infty}\left(\nu^{(a)}\right) \alpha_{a}\right) .
$$

It does not depend on the rigging $J$.

Example 2.2. The following diagrams show an example of rigged configuration of weight $6 \varpi_{1}+4 \varpi_{2}-\left(4 \alpha_{1}+4 \alpha_{2}+\alpha_{3}\right)$.

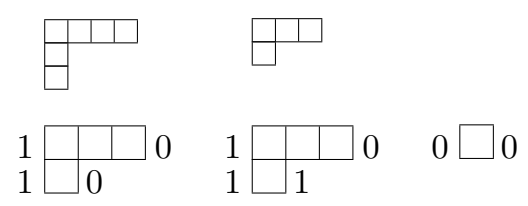

Upper diagrams are $L^{(1)}, L^{(2)}, L^{(3)}\left(L^{(3)}\right.$ is empty) drawn from left to right and lower ones are $\nu^{(1)}, \nu^{(2)}, \nu^{(3)}$. On the left (resp. right) of each row the corresponding vacancy number (resp. rigging) is written.

\section{KSS BIJECTION AND SIMILARITY}

3.1. Operations on paths. We define several operations on paths. We first define $\mathrm{lh}, \mathrm{lb}^{(s)}, \mathrm{ls}^{(m)}$. In this subsection $B$ is a tensor product of KR crystals.

(1) Suppose $B=B^{1,1} \otimes B^{\prime}, b=c \otimes b^{\prime} \in B^{1,1} \otimes B^{\prime}$. The map lh $: B \rightarrow B^{\prime}$ is defined by $\operatorname{lh}(b)=b^{\prime}$. We set $\ln (B)=B^{\prime}$.

(2) Suppose $B=B^{r, s} \otimes B^{\prime}, b=c \otimes b^{\prime} \in B^{r, s} \otimes B^{\prime}(r \geq 2)$. The map $\mathrm{lb}^{(s)}$ : $B \rightarrow B^{1, s} \otimes B^{r-1, s} \otimes B^{\prime}$ is defined by $\mathrm{lb}^{(s)}(b)=c^{\prime} \otimes c^{\prime \prime} \otimes b^{\prime}$, where $c^{\prime}$ is the lowest row of $c$ and $c^{\prime \prime}$ is obtained by removing $c^{\prime}$ from $c$. We set $\mathrm{lb}^{(s)}(B)=B^{1, s} \otimes B^{r-1, s} \otimes B^{\prime}$.

(3) Suppose $B=B^{r, s} \otimes B^{\prime}, b=c \otimes b^{\prime} \in B^{r, s} \otimes B^{\prime}(s \geq 2)$. The map ls ${ }^{(m)}$ : $B \rightarrow B^{r, m} \otimes B^{r, s-m} \otimes B^{\prime}(1 \leq m<s)$ is defined by ls ${ }^{(m)}(b)=c^{\prime} \otimes c^{\prime \prime} \otimes b^{\prime}$, where $c^{\prime}$ is the leftmost $m$ columns of $c$ and $c^{\prime \prime}$ is obtained by removing $c^{\prime}$ from $c$. We set ls ${ }^{(s)}(B)=B^{r, m} \otimes B^{r, s-m} \otimes B^{\prime}$.

These maps send a path to another path. In [20] operations $\mathrm{lb}^{(s)}, \mathrm{ls}^{(m)}$ were defined only when $s=1$ and $m=1$. We need the extensions of them to prove our main result. We set $l b=l^{(1)}$, ls $=l_{s}^{(1)}$.

Example 3.1. For a path $b$ in Example 2.1 we have $b^{\prime}=\operatorname{ls}(b), b^{\prime \prime}=\operatorname{lb}\left(b^{\prime}\right), b^{\prime \prime \prime}=$ $\operatorname{lh}\left(b^{\prime \prime}\right)$ as follows.

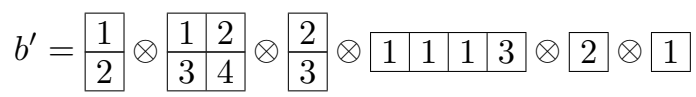

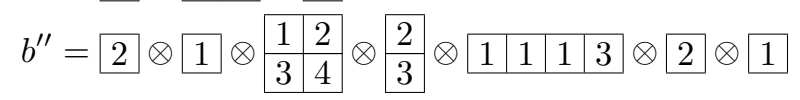

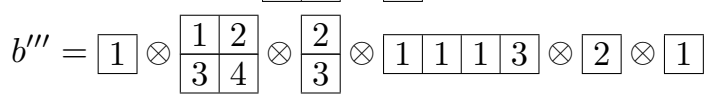


For later use we need the transpose of a path $b$ given in [20]. Let $B=B^{r_{k}, s_{k}} \otimes$ $B^{r_{k-1}, s_{k-1}} \otimes \cdots \otimes B^{r_{1}, s_{1}}$. For $b=b_{k} \otimes b_{k-1} \otimes \cdots \otimes b_{1} \in B$ rotate each rectangular tableau $b_{i}$ by $90^{\circ}$ clockwise to obtain $\tilde{b}_{i}$. Suppose the letter $a$ occurs in cell $c$ of $\tilde{b}_{i}$. Then replace letter $a$ in cell $c$ by $\tilde{a}$ where $\tilde{a}$ is chosen such that the letter $a$ in cell $c$ is the $\tilde{a}$-th letter $a$ in $\operatorname{row}(b)$ reading from right to left. Finally, turn each tableau up side down and define it to be $\operatorname{tr}(b)$. If $b \in \mathcal{P}(B)$, then $\operatorname{tr}(b) \in \mathcal{P}(\operatorname{tr}(B))$ where $\operatorname{tr}(B)=B^{s_{k}, r_{k}} \otimes B^{s_{k-1}, r_{k-1}} \otimes \cdots \otimes B^{s_{1}, r_{1}}$. The map tr satisfies $\operatorname{tr}^{2}=\mathrm{id}$.

Example 3.2. For a path $b$ in Example 2.1 we have

$$
\operatorname{tr}(b)=\begin{array}{|l|l|}
\hline 1 & 3 \\
\hline 3 & 5 \\
\hline 4 & 6 \\
\hline
\end{array} \otimes 2 \begin{array}{l|l|}
2 & 2
\end{array} \otimes \begin{array}{|l|}
\hline \frac{1}{2} \\
\hline 3 \\
\hline 4
\end{array} \otimes 11 \otimes 1 .
$$

Finally, we define the map $S_{m}$ on $\mathcal{P}(B)$. For an element $b=b_{k} \otimes b_{k-1} \otimes \cdots \otimes b_{1}$ of $B$ we define $S_{m}(b)=S_{m}\left(b_{k}\right) \otimes S_{m}\left(b_{k-1}\right) \otimes \cdots \otimes S_{m}\left(b_{1}\right)$, where $S_{m}$ on a single KR crystal was defined in $\S 2.1$. If $b \in \mathcal{P}(B)$, then we have $S_{m}(b) \in \mathcal{P}\left(S_{m}(B)\right)$, where $S_{m}(B)$ is obtained by replacing each single KR crystal $B^{r, s}$ in $B$ with $B^{r, m s}$. It is easy to see that $S_{m} \circ S_{m^{\prime}}=S_{m m^{\prime}}$.

Example 3.3. For a path $b$ in Example 2.1 we have

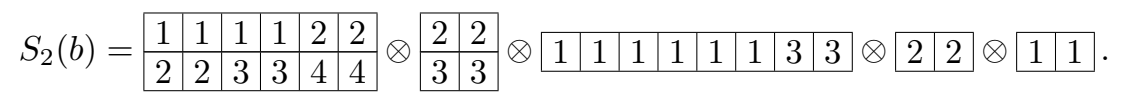

3.2. Operations on rigged configurations. We define the corresponding operations on rigged configurations. We first define $\delta, \beta^{(s)}, \gamma^{(m)}$. Say a row of a rigged configuration singular if its rigging is equal to the vacancy number $p_{i}^{(a)}$.

(1) Suppose $L^{(1)}$ contains a row of length 1 . Set $\ell^{(0)}=1$ and repeat the following process for $a=1,2, \ldots, n$ or until stopped. Find the smallest integer $i \geq \ell^{(a-1)}$ such that there exists a singular row of length $i$ in $(\nu, J)^{(a)}$. If no such $i$ exists, set $\operatorname{rk}(\nu, J)=a$ and stop. Otherwise, select a row of length $i$, set $\ell^{(a)}=i$ and continue the process with $a+1$. Set all undefined $\ell^{(a)}$ to $\infty$. As for the new multiplicity array $\tilde{L}, \tilde{L}^{(1)}$ is given by removing a row of length 1 from $L^{(1)}$ and other $L^{(a)}$ remain the same. $\delta(\nu, J)$ is obtained by removing a box from the selected rows and making the new rows singular again.

(2) Suppose $L^{(r)}$ contains a row of length $s$. $\tilde{L}$ is given by removing a row of length $s$ from $L^{(r)}$ and adding a row of length $s$ to both $L^{(1)}$ and $L^{(r-1)}$ (two rows of length $s$ to $L^{(1)}$ if $r=2$ ). $\beta^{(s)}(\nu, J)$ is obtained by adding singular rows of length $s$ to $(\nu, J)^{(a)}$ for $1 \leq a<r$.

(3) Suppose $L^{(r)}$ contains a row of length $s$. $\tilde{L}$ is given by removing a row of length $s$ from $L^{(r)}$ and adding a row of length $m$ and a row of length $s-m$. We set $\gamma^{(m)}(\nu, J)=(\nu, J)$.

These maps send a rigged configuration in $\mathcal{R C}(L)$ to another one in $\mathcal{R C}(\tilde{L})$. In [20] operations $\beta^{(1)}, \gamma^{(1)}$ were defined in the notations $j, i$. We set $\beta=\beta^{(1)}, \gamma=\gamma^{(1)}$.

Example 3.4. Let $(\nu, J)$ be as in Example 2.2. Then $\left(\nu^{\prime}, J^{\prime}\right)=\gamma(\nu, J),\left(\nu^{\prime \prime}, J^{\prime \prime}\right)=$ $\beta\left(\nu^{\prime}, J^{\prime}\right),\left(\nu^{\prime \prime \prime}, J^{\prime \prime \prime}\right)=\delta\left(\nu^{\prime \prime}, J^{\prime \prime}\right)$ are given successively as follows. 

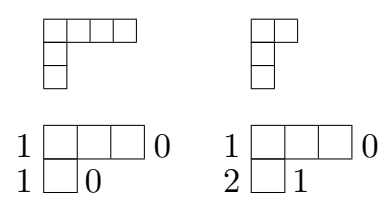

$0 \square 0$
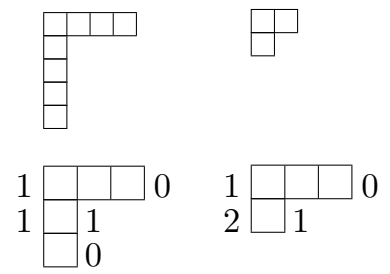

$0 \square 0$
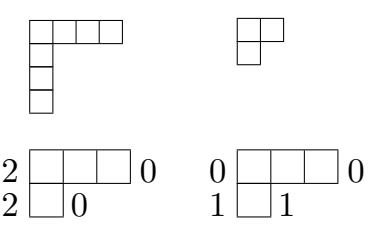

$0 \square 0$

Example 3.5. In the next example $L^{(1)}=\left(1^{13}\right), L^{(2)}=L^{(3)}=\emptyset$. $\delta$ removes the box with $\times$.
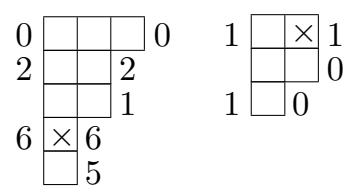

\begin{tabular}{l|l|l}
\hline & $\times$ & 0 \\
0 & 0
\end{tabular}

$0 \square 0$

Next we define the map tr on rigged configurations following [20] (see also [11]). The new multiplicity array $\tilde{L}=\left(\tilde{L}_{i}^{(a)}\right)$ is defined by $\tilde{L}_{i}^{(a)}=L_{a}^{(i)}$. We assume $n$ is sufficiently large so that $\tilde{L}$ is well defined. For a configuration $\nu=\left(m_{i}^{(a)}\right)$ define a matrix $N=\left(N_{a i}\right)_{a \in I_{0}, i \in \mathbb{Z}_{>0}}$ by

$$
N_{a i}=\sum_{j \geq i}\left(m_{j}^{(a-1)}-m_{j}^{(a)}\right) .
$$

Note that $\sum_{j \geq i} m_{i}^{(a)}$ is the depth of the $i$-th column of $\nu^{(a)} \cdot m_{j}^{(0)}$ is defined to be zero for any $j$. Now set $(\tilde{\nu}, \tilde{J})=\operatorname{tr}(\nu, J)$. Then $\tilde{N}=N(\tilde{\nu})$ is given by

$$
\tilde{N}_{i a}=-N_{a i}+\chi((a, i) \in \lambda)-\sum_{b, j} L_{j}^{(b)} \chi(a \leq b \& i \leq j) .
$$

Here $\chi(\theta)=1$ if $\theta$ is true, $=0$ otherwise. $\lambda$ is the partition $\left(\lambda_{1}, \lambda_{2}, \ldots\right)$ such that the weight of $(\nu, J)$ is given by $\sum_{j} \lambda_{j}\left(\varpi_{j}-\varpi_{j-1}\right)\left(\varpi_{0}=0\right)$, and $(a, i) \in \lambda$ signifies that the cell of the $a$-th row and the $i$-th column belongs to the Young diagram of $\lambda$.

Note that the rigging $J$ can be viewed as a double sequence of Young diagrams $J=\left(J^{(a, i)}\right)$ where $J^{(a, i)}$ is a Young diagram inside the rectangle of depth $m_{i}^{(a)}$ and 
width $p_{i}^{(a)}$. The Young diagram $\tilde{J}^{(i, a)}$ is defined as the transpose of the complementary Young diagram to $J^{(a, i)}$ in the rectangle of depth $m_{i}^{(a)}$ and width $p_{i}^{(a)}$. This $\operatorname{tr}$ also satisfies $\operatorname{tr}^{2}=\mathrm{id}$. The following lemma, which is clear from the above rule, is used later.

Lemma 3.1. Let $B^{r, s}$ be the leftmost tensor factor of $B$. Then we have $\mathrm{ls} \circ \mathrm{tr}=$ $\operatorname{tr} \circ \mathrm{lb}^{(s)}$ on $\mathcal{P}(B)$.

Example 3.6. Let $(\nu, J)$ be as in Example 2.2. Then we have

$$
\lambda=\lambda, \quad N=\left(\begin{array}{ccc}
-2 & -1 & -1 \\
0 & 0 & 0 \\
1 & 1 & 1 \\
1 & 0 & 0
\end{array}\right), \quad \tilde{N}=\left(\begin{array}{cc}
-2 & -1 \\
0 & 0 \\
0 & 0 \\
0 & 1 \\
1 & 0 \\
1 & 0
\end{array}\right) \text {. }
$$

All entries of $N, \tilde{N}$ outside the given part are zero. Thus $\operatorname{tr}(\nu, J)$ is given as follows.
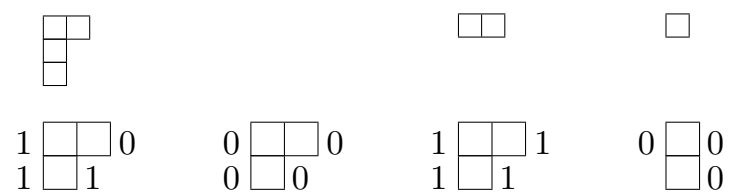

$0 \square 0$

Finally, operation $S_{m}$ on rigged configurations is defined by enlarging all rows of $L^{(a)}$ and $\nu^{(a)} m$ times and multiplying $m$ to all riggings. Note that $p_{m i}^{(a)}\left(S_{m}(\nu)\right)=$ $m p_{i}^{(a)}(\nu)$. It is again easy to see that $S_{m} \circ S_{m^{\prime}}=S_{m m^{\prime}}$.

Example 3.7. Let $(\nu, J)$ be as in Example 2.2. $S_{2}(\nu, J)$ is given below.
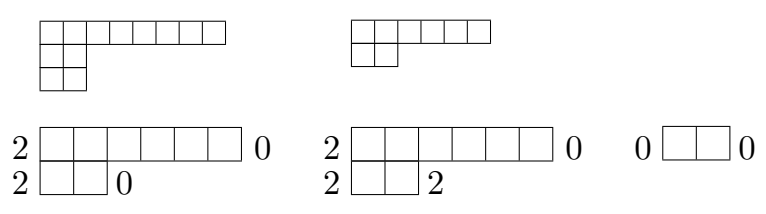

3.3. KSS bijection and the main theorem. For a tensor product of KR crystals $B=B^{r_{k}, s_{k}} \otimes B^{r_{k-1}, s_{k-1}} \otimes \cdots \otimes B^{r_{1}, s_{1}}$ we define the corresponding multiplicity array $L(B)=\left(L_{i}^{(a)}\right)$ by

$$
L_{i}^{(a)}=\sharp\left\{j \mid\left(r_{j}, s_{j}\right)=(a, i), 1 \leq j \leq k\right\} .
$$

The following theorem is proved essentially in [11]. We adopt the formulation in $[20]$.

Theorem 3.2. Let $B=B^{1,1} \otimes B^{\prime}$ in (1), $B=B^{r, 1} \otimes B^{\prime}(r \geq 2)$ in (2), and $B=B^{r, s} \otimes B^{\prime}(s \geq 2)$ in (3). There exists a unique bijection $\Phi$ from $\mathcal{P}(B)$ to $\mathcal{R C}(L(B))$ that maps the empty path to the empty rigged configurations and makes 
the following diagrams commutative.

(1)

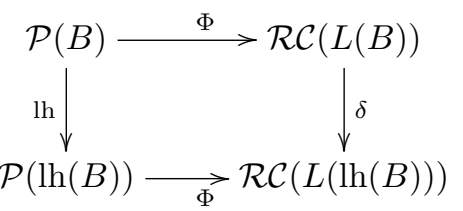

(3)

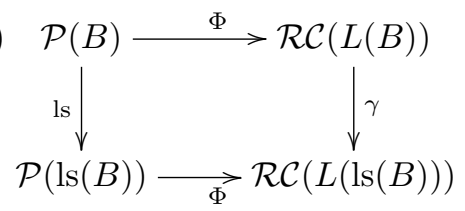

$(2)$

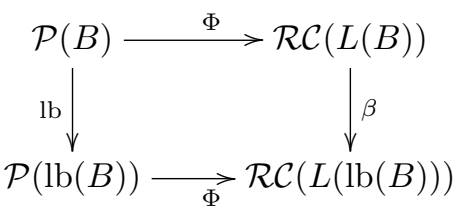

Moreover, under $\Phi$, tr corresponds to tr and $R_{i}$ corresponds to id for any $i=$ $1,2, \ldots, k-1$ where $R_{i}$ stands for the combinatorial $R$-matrix acting on the $(i+1)$ th and the $i$-th position $B_{i+1} \otimes B_{i}$ of $B$.

Now we can state our theorem.

Theorem 3.3. Under $\Phi, S_{m}$ corresponds to $S_{m}$.

We need to prepare lemmas to prove it.

Lemma 3.4. Suppose $(\nu, J) \in \mathcal{R C}(L)$ with $L_{1}^{(1)}>0$. We apply $\delta$ on $(\nu, J)$. Let $\ell^{(1)} \leq \ell^{(2)} \leq \cdots \leq \infty$ be the lengths of rows whose box is removed from $\nu^{(1)}, \nu^{(2)}, \ldots$ by $\delta$ and $\operatorname{set}(\tilde{\nu}, \overline{\tilde{J}})=\delta(\nu, J)$. Then we have

$$
p_{i}^{(a)}(\tilde{\nu})=p_{i}^{(a)}(\nu)-\chi\left(\ell^{(a-1)} \leq i\right)+2 \chi\left(\ell^{(a)} \leq i\right)-\chi\left(\ell^{(a+1)} \leq i\right) .
$$

Proof. Easy from (2.9).

Lemma 3.5. Suppose $(\nu, J) \in \mathcal{R C}(L)$ with $L_{1}^{(1)}>0$. Let $\left(\nu^{\prime}, J^{\prime}\right)=S_{m}(\nu, J)$. Then we can apply $\delta \circ(\delta \circ \gamma)^{m-1}$ on $\left(\nu^{\prime}, J^{\prime}\right)$. During the operation $m$ boxes are removed from the same row in each $\nu^{\prime(a)}$.

Proof. Let $\ell^{(1)}, \ell^{(2)}, \ldots$ be as in the previous lemma. Since a singular row remains singular and a nonsingular row does nonsingular by $S_{m}$, by the first $\delta \circ \gamma$ a box is removed from a row of length $m \ell^{(a)}$ in each $\nu^{\prime(a)}$.

Now we apply next $\delta \circ \gamma$ on $(\tilde{\nu}, \tilde{J})=(\delta \circ \gamma)\left(\nu^{\prime}, J^{\prime}\right)$. We show that during this process a box is removed from a singular row in $\tilde{\nu}^{(a)}$ of length $m \ell^{(a)}-1$ proceeding with $a=1,2, \ldots$. Suppose $\ell^{(a)}<\infty$. From the previous lemma, we have

$$
p_{i}^{(a)}(\tilde{\nu})-p_{i}^{(a)}\left(\nu^{\prime}\right)= \begin{cases}0 & \text { if } i<m \ell^{(a-1)} \\ -1 & \text { if } m \ell^{(a-1)} \leq i<m \ell^{(a)} .\end{cases}
$$

Here $\ell^{(0)}$ should be understood as $1 / m$. We look for a singular row in $\tilde{\nu}^{(a)}$ of length not less than $m \ell^{(a-1)}-1$. Suppose $\ell^{(a-1)}<\ell^{(a)}$. In the interval $i<m \ell^{(a-1)}$ we only need to consider the case of $i=m \ell^{(a-1)}-1$. However, there is no row in $\tilde{\nu}^{(a)}$ of this length. In the interval $m \ell^{(a-1)} \leq i<m \ell^{(a)}$ we have $p_{i}^{(a)}(\tilde{\nu})=p_{i}^{(a)}\left(\nu^{\prime}\right)-1$. However, all the riggings in $\tilde{\nu}^{(a)}$ are multiple of $m$ and hence remain nonsingular, except the one in the row of length $m \ell^{(a)}-1$ from which a box is removed in the previous $\delta$. Since this row is singular, we remove a box from it. When $\ell^{(a-1)}=\ell^{(a)}$, from the same reason a box is removed from a unique row of length $m \ell^{(a)}-1$. Suppose now $\ell^{(a)}=\infty$. Then all rows of length not less than $m \ell^{(a-1)}-1$ are nonsingular. 
We continue the application of $\delta(\circ \gamma)$ but the fact that a box is removed from the same row in each $\nu^{\prime(a)}$ remains true.

Proposition 3.6. Suppose the leftmost factor of $B$ is $B^{r, s}$. Then the following diagram commutes.

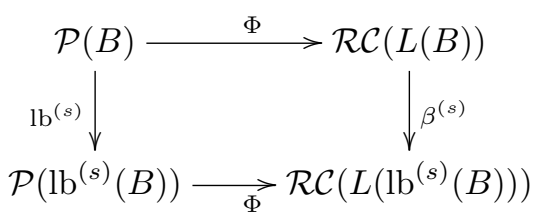

Proof. First recall Lemma 5.3 given in [11]. Consider the following diagram.

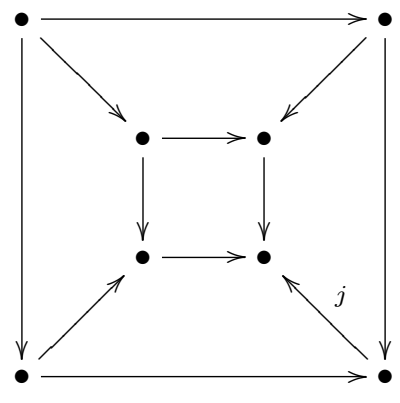

Viewing this diagram as a cube with front face given by the large square, suppose the square diagrams given by all faces of the cube except the front commute. Assume also that the map $j$ is injective. Then the front face should also commute.

Now consider the following diagram.

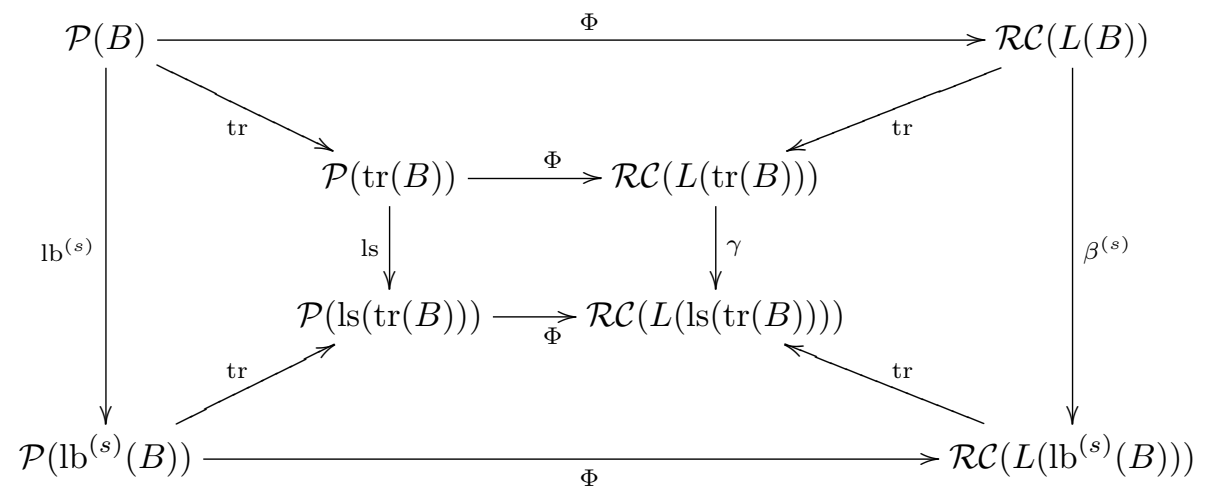

We wish to show the front face commutes. The left face commutes by Lemma 3.1 and the back, top, bottom faces do by Theorem 3.2. Since tr is a bijection, we are left to show the right face commutes. Let $\beta^{(s)}(\nu, J)=\left(\nu^{\prime}, J^{\prime}\right)$, and $\operatorname{tr}(\nu, J)=$ $(\tilde{\nu}, \tilde{J}), \operatorname{tr}\left(\nu^{\prime}, J^{\prime}\right)=\left(\tilde{\nu}^{\prime}, \tilde{J}^{\prime}\right)$. In view of the description of $\gamma$, it is enough to show $\tilde{\nu}=\tilde{\nu}^{\prime}, \tilde{J}=\tilde{J}^{\prime}$.

We first prove $\tilde{\nu}=\tilde{\nu}^{\prime}$. Let $m_{j}^{(a)}$ be defined as (2.7) for $\nu$ and $m_{j}^{(a)}$ for $\nu^{\prime}$. Then we have

$$
m_{j}^{\prime(a)}=m_{j}^{(a)}+\chi(a<r) \delta_{j s}
$$


Using a similar notation for $N_{a i}$ in (3.1) to $\nu$ we get

$$
N_{a i}^{\prime}=N_{a i}+\left(\delta_{a r}-\delta_{a 1}\right) \chi(i \leq s) .
$$

Then by (3.2) one calculates

$$
\begin{aligned}
\tilde{N}_{i a}^{\prime}= & -N_{a i}^{\prime}+\chi((a, i) \in \lambda)-\sum_{b, j} L_{j}^{\prime(b)} \chi(a \leq b \& i \leq j) \\
= & -N_{a i}-\left(\delta_{a r}-\delta_{a 1}\right) \chi(i \leq s)+\chi((a, i) \in \lambda) \\
& \quad-\sum_{b, j}\left(L_{j}^{(b)}+\left(\delta_{b, r-1}+\delta_{b 1}-\delta_{b r}\right) \delta_{j s}\right) \chi(a \leq b \& i \leq j) \\
= & \tilde{N}_{i a}
\end{aligned}
$$

and hence we obtain $\tilde{\nu}=\tilde{\nu}^{\prime}$.

To prove $\tilde{J}=\tilde{J}^{\prime}$ note that $J^{(a, i)}=J^{\prime(a, i)}$ except when $a<r$ and $i=s$, in which case $J^{\prime(a, i)}$ has an extra row of length $p_{i}^{(a)}$ compared to $J^{(a, i)}$. Recalling $p_{i}^{(a)}=p_{i}^{(a)}$ and that the partition corresponding to tr is defined as the transpose of the complementary partition in the rectangle of height $m_{i}^{(a)}$ and width $p_{i}^{(a)}$, we obtain $\tilde{J}=\tilde{J}^{\prime}$.

Proposition 3.7. Let $b \in B^{r, s}$ and $1 \leq m<s$. Then we have

$$
R\left(\mathrm{ls}^{(m)}(b)\right)=\mathrm{ls}^{(s-m)}(b) .
$$

Proof. It is clear from the combinatorial description of $R$ in $\S 2.2$.

Proposition 3.8. Suppose the leftmost factor of $B$ is $B^{r, s}$ with $s \geq 2$. For any $m$ $(1 \leq m<s)$ the following diagram commutes.

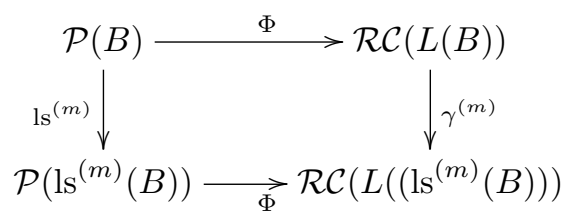

Proof. We prove by induction on $m$. The $m=1$ case is nothing but Theorem 3.2 (3). Consider the following sequence of maps.

$$
\begin{aligned}
& B^{r, s} \otimes B^{\prime} \stackrel{\mathrm{ls}^{(m-1)}}{\longrightarrow} B^{r, m-1} \otimes B^{r, s-m+1} \otimes B^{\prime} \stackrel{R \otimes 1}{\longrightarrow} B^{r, s-m+1} \otimes B^{r, m-1} \otimes B^{\prime} \\
& \stackrel{\mathrm{ls}}{\longrightarrow} B^{r, 1} \otimes B^{r, s-m} \otimes B^{r, m-1} \otimes B^{\prime} \stackrel{1 \otimes R \otimes 1}{\longrightarrow} B^{r, 1} \otimes B^{r, m-1} \otimes B^{r, s-m} \otimes B^{\prime} \\
& \stackrel{\mathrm{ls}-1}{\longrightarrow} B^{r, m} \otimes B^{r, s-m} \otimes B^{\prime}
\end{aligned}
$$

At each step the maps cut, move the cutting line, or concatenate the tableau belonging to $B^{r, s}$, by the definition of $\mathrm{ls}^{(k)}$ and Proposition 3.7. In particular, the last map $l^{-1}$ is well defined. It is clear that the composition of these maps coincides with $\mathrm{ls}^{(m)}$. Since all maps in the sequence correspond to the identity in the rigged configuration side by the induction hypothesis and Theorem 3.2, the proof is finished.

Proof of Theorem 3.3. For $B=B^{r_{k}, s_{k}} \otimes B^{r_{k-1}, s_{k-1}} \otimes \cdots \otimes B^{r_{1}, s_{1}}$ we introduce $\left(\sum_{j=1}^{k} r_{j} s_{j}, \sum_{j=1}^{k}\left(r_{j}-1\right) s_{j}, \sum_{j=1}^{k}\left(s_{j}-1\right)\right)$. Note that the operation for $B$ in $(\mathrm{i})(i=$ $1,2,3)$ of Theorem 3.2 decreases its $i$-th component by 1 . We prove by induction 
on the lexicographic order of this index. We again use the cube diagram introduced in the proof of Proposition 3.6.

First suppose that the leftmost factor of $B$ is $B^{1,1}$ and consider the following diagram.

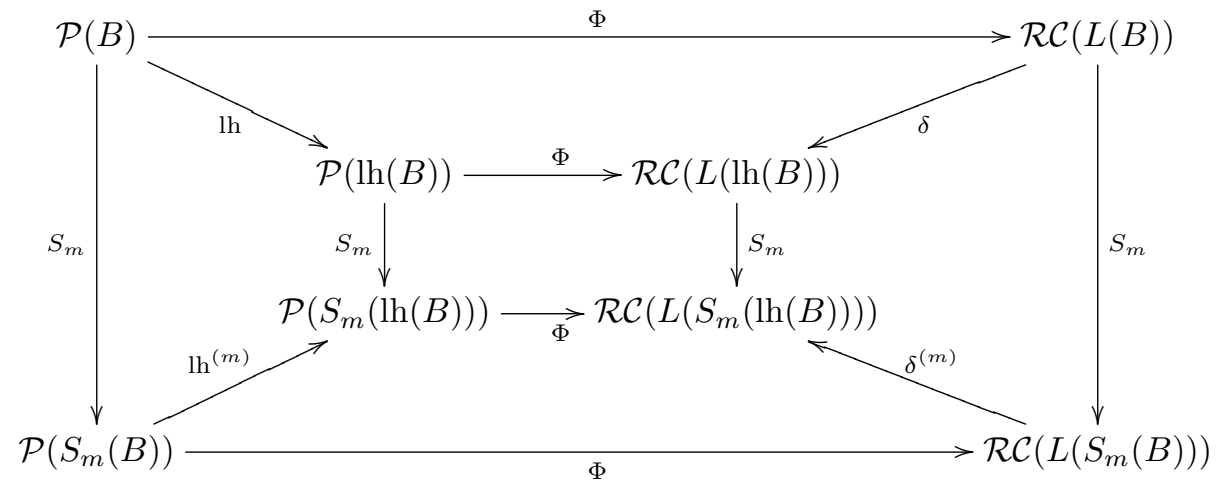

Here we have set $\mathrm{lh}^{(m)}=\mathrm{lh} \circ(\mathrm{lh} \circ \mathrm{ls})^{m-1}, \delta^{(m)}=\delta \circ(\delta \circ \gamma)^{m-1}$. We wish to show the front face commutes. Note that if we restrict the domain to that of a fixed weight, $\delta^{(m)}$ is injective. By the above lemma and the injectivity, it is enough to show all the other faces commute. The back face is assumed to commute by induction. The commutativity of the left face is clear, while the right face is due to Lemma 3.5. The top and bottom faces commute by Theorem 3.2 .

Next suppose that the leftmost factor of $B$ is $B^{r, 1}(r \geq 2)$. Consider

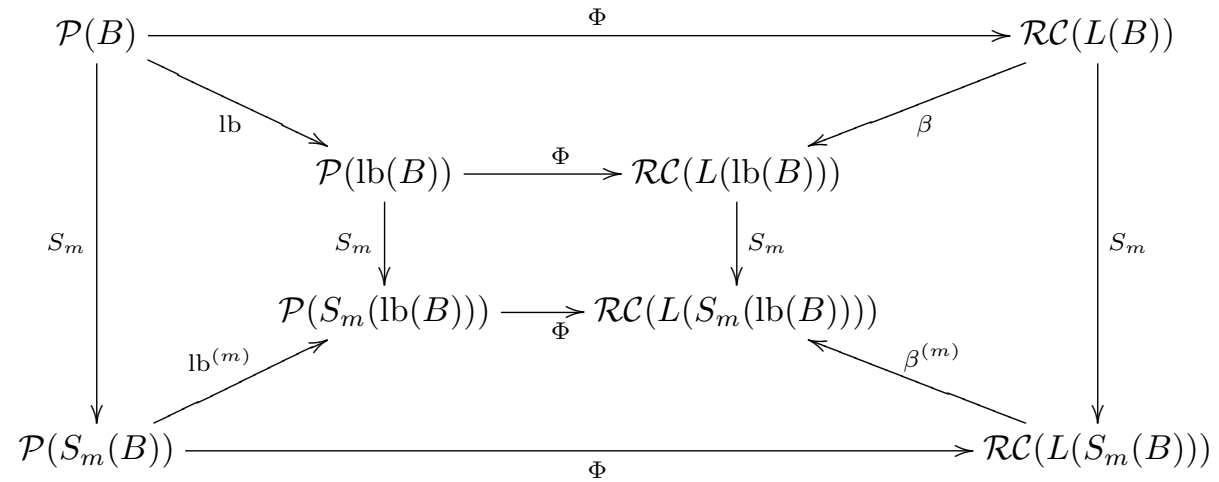

Again, we show all the other faces other than the front one commute. The back face commutes by induction. The commutativity of the left and right faces is clear. The top face commutes by Theorem 3.2, while the bottom face is due to Proposition 3.6 . 
Finally suppose that the leftmost factor of $B$ is $B^{r, s}(s \geq 2)$. Consider

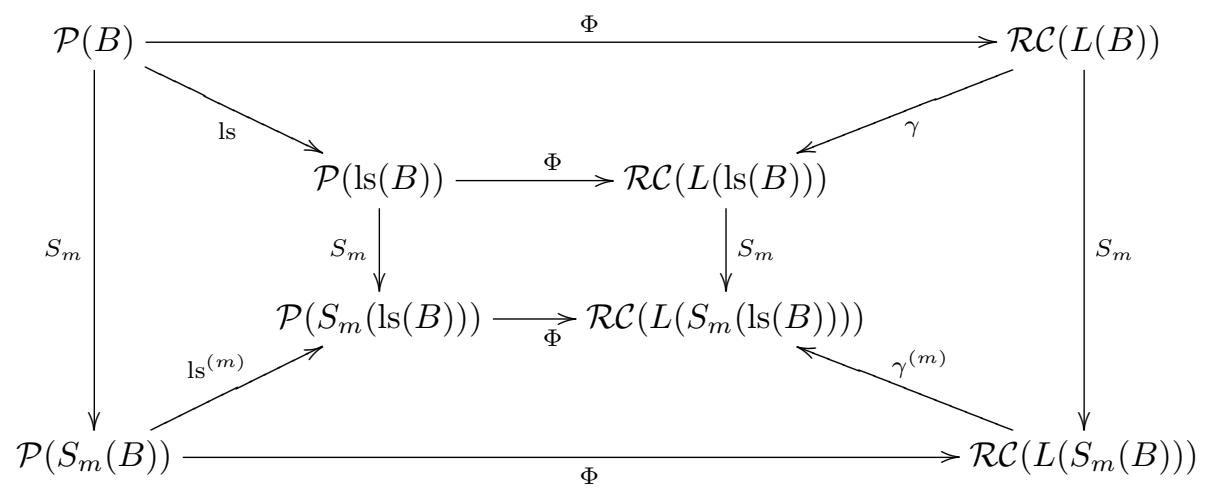

In this case we use Proposition 3.8 for the commutativity of the bottom face.

\section{Application}

Theorem 3.3 motivates us to extend the notions of $\mathcal{P}(B)$ and $\mathcal{R C}(L)$. In the sequel we assume $R$ to be one of $\mathbb{Z}, \mathbb{Q}$ or $\mathbb{R}$. We set $R_{>0}=\{z \in R \mid z>0\}, R_{\geq 0}=$ $\{z \in R \mid z \geq 0\}$. First we recall an alternative description of $B^{r, s}$. For a tableau in $B^{r, s}$ let $x_{i, j}(1 \leq i \leq r, i \leq j \leq n-r+i+1)$ be the number of boxes with letter $j$ in the $i$-th row. Then they must satisfy

$$
\begin{aligned}
& \sum_{k=i}^{n-r+i+1} x_{i, k}=s \quad(i=1,2, \ldots, r), \\
& \sum_{k=i}^{j} x_{i, k} \geq \sum_{k=i+1}^{j+1} x_{i+1, k} \quad(i=1, \ldots, r-1 ; j=i, \ldots, n-r+i) .
\end{aligned}
$$

Now for $r \in I_{0}, s \in R_{>0}$ define

$$
\mathcal{B}^{r, s}=\left\{\left(x_{i, j}\right) \mid x_{i, j} \in R_{\geq 0} \text { and satisfies (4.1) and (4.2) }\right\} .
$$

We can also consider a formal tensor product

$$
\mathcal{B}=\mathcal{B}^{r_{k}, s_{k}} \otimes \cdots \otimes \mathcal{B}^{r_{1}, s_{1}} .
$$

Recall that $\mathcal{P}(B)$ was defined as the set of elements $b$ satisfying $e_{i} b=0$ for $i \in I_{0}$ in $\S 2.2$. Note that $e_{i} b=0$ is equivalent to $\varepsilon_{i}(b)=0$. Hence our new notion $\mathcal{P}_{R}(\mathcal{B})$ should be defined as the set of elements $b \in \mathcal{B}$ satisfying $\varepsilon_{i}(b)=0$ for $i \in I_{0}$ where $\varepsilon_{i}(b)$ for $b \in \mathcal{B}^{r, s}$ is given in $[19, \S 5.2]$ and $\varepsilon_{i}(b)$ for a multiple tensor product $b \in \mathcal{B}$ is calculated by extending (2.3) via coassociativity. For $m \in R_{>0}$ the similarity map $S_{m}$ on $\mathcal{P}_{R}(\mathcal{B})$ is defined as follows. For $b=b_{k} \otimes \cdots \otimes b_{1}, b_{l}=\left(x_{i, j}^{(l)}\right) \in \mathcal{B}^{r_{l}, s_{l}}$, $S_{m}(b)=b_{k}^{\prime} \otimes \cdots \otimes b_{1}^{\prime}$ is given by $b_{l}^{\prime}=\left(m x_{i, j}^{(l)}\right)$ for $1 \leq l \leq k$. Then $S_{m}$ turns out a map from $\mathcal{P}_{R}(\mathcal{B})$ to $\mathcal{P}_{R}\left(\mathcal{B}^{\prime}\right)$ where $\mathcal{B}^{\prime}=\mathcal{B}^{r_{k}, m s_{k}} \otimes \cdots \otimes \mathcal{B}^{r_{1}, m s_{1}}$. It is easy to see that $S_{m} \circ S_{m^{\prime}}=S_{m m^{\prime}}$.

The set of rigged configurations $\mathcal{R C}(L)$ defined in $\S 2.3$ can also be extended by allowing the indices $i$ to take values in $R_{>0}$. Consider $L=\left(L_{i}^{(a)}\right)_{a \in I_{0}, i \in R_{>0}}$ such that $L_{i}^{(a)} \in \mathbb{Z}_{\geq 0}$ and $L_{i}^{(a)}>0$ only for finitely many $(a, i)$. Let $\nu=\left(m_{i}^{(a)}\right)_{a \in I_{0}, i \in R_{>0}}$ be similar such data. $\nu$ is said to be an admissible configuration if it satisfies

$$
p_{i}^{(a)} \geq 0 \quad \text { for any } a \in I_{0} \text { and } i \in R_{>0},
$$


where

$$
p_{i}^{(a)}=\sum_{j \in R_{>0}}\left(L_{j}^{(a)} \min (i, j)-\sum_{b \in I_{0}} C_{a b} \min (i, j) m_{j}^{(b)}\right) .
$$

We identify an admissible configuration $\nu$ with a sequence $\left(\nu^{(a)}\right)_{a \in I_{0}}$ such that each $\nu^{(a)}$ is a weakly decreasing sequence of elements of $R_{>0}$ in which every $i \in R_{>0}$ appears $m_{i}^{(a)}$ times. $L^{(a)}=\left(L_{i}^{(a)}\right)_{i \in R_{>0}}$ and $\nu^{(a)}$ can be viewed as a generalized Young diagram in which each row can have length in the set $R_{>0}$. A rigging $J$ on $\nu$ is to associate, with each part of such generalized Young diagram $\nu^{(a)}$ of the same width $i$, a sequence $\left(J_{1}^{(a, i)} \geq J_{2}^{(a, i)} \geq \ldots \geq J_{m_{i}^{(a)}}^{(a, i)}\right)$ of length $m_{i}^{(a)}$ such that $J_{j}^{(a, i)} \in R_{\geq 0}$ for $j=1, \ldots, m_{i}^{(a)}$ and $p_{i}^{(a)} \geq J_{1}^{(a, i)}$. We call such pair $(\nu, J)$ a rigged configuration and denote the set of rigged configurations by $\mathcal{R C}_{R}(L)$. For $m \in R_{>0}$ the similarity map $S_{m}$ on $\mathcal{R C}_{R}(L)$ is defined by enlarging all rows of $L^{(a)}$ and $\nu^{(a)} m$ times and multiplying $m$ to all riggings. It is again easy to see that $S_{m} \circ S_{m^{\prime}}=S_{m m^{\prime}}$.

For $\mathcal{B}=\mathcal{B}^{r_{k}, s_{k}} \otimes \cdots \otimes \mathcal{B}^{r_{1}, s_{1}}$ we define $L(\mathcal{B})=\left(L_{i}^{(a)}\right)_{a \in I_{0}, i \in R_{>0}}$ by (3.3). From Theorem 3.2 there is a bijection $\Phi_{\mathbb{Z}}=\Phi$ from $\mathcal{P}_{\mathbb{Z}}(\mathcal{B})$ to $\mathcal{R C}_{\mathbb{Z}}(L(\mathcal{B}))$. We now define a map $\Phi_{\mathbb{Q}}$ from $\mathcal{P}_{\mathbb{Q}}(\mathcal{B})$ to $\mathcal{R C}_{\mathbb{Q}}(L(\mathcal{B}))$. Let $b=b_{k} \otimes \cdots \otimes b_{1}, b_{l}=\left(x_{i, j}^{(l)}\right) \in \mathcal{B}^{r_{l}, s_{l}}$ be an element of $\mathcal{P}_{\mathbb{Q}}(\mathcal{B})$. Take the minimal positive integer $m_{0}$ such that $m_{0} x_{i j}^{(l)} \in \mathbb{Z}$ for all $i, j, l$. We define $\Phi_{\mathbb{Q}}(b)=\left(S_{1 / m_{0}} \circ \Phi \circ S_{m_{0}}\right)(b)$.

Proposition 4.1. This $\Phi_{\mathbb{Q}}$ is a bijection from $\mathcal{P}_{\mathbb{Q}}(\mathcal{B})$ to $\mathcal{R} \mathcal{C}_{\mathbb{Q}}(L(\mathcal{B}))$.

Proof. For well-definedness we need to show that if $m$ is a multiple of $m_{0}$, the image of the above map $\Phi_{\mathbb{Q}}$ stays the same with $m_{0}$ replaced with $m$. But it is true since all the small diagrams below are commutative. (The middle one is from Theorem 3.3.)

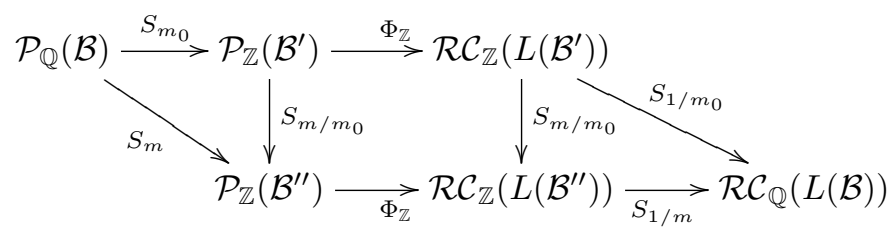

where $\mathcal{B}^{\prime}=\mathcal{B}^{r_{k}, m_{0} s_{k}} \otimes \cdots \otimes \mathcal{B}^{r_{1}, m_{0} s_{1}}, \mathcal{B}^{\prime \prime}=\mathcal{B}^{r_{k}, m s_{k}} \otimes \cdots \otimes \mathcal{B}^{r_{1}, m s_{1}}$.

Similarly, we can try to define a map $\Phi_{\mathbb{R}}$ from $\mathcal{P}_{\mathbb{R}}(\mathcal{B})$ to $\mathcal{R C}_{\mathbb{R}}(L(\mathcal{B}))$ by using the similarity map $S_{m}$ for $m \in \mathbb{R}$ and considering a sequence of elements in $\mathcal{P}_{\mathbb{Q}}\left(\mathcal{B}^{\prime}\right)$ convergent to the element in $\mathcal{P}_{\mathbb{R}}(\mathcal{B})$. (The second index of each single $\mathrm{KR}$ crystal in $\mathcal{B}^{\prime}$ is slightly shifted from $\mathcal{B}$.) We conjecture that this map is well-defined, but its proof seems nontrivial. We also conjecture that $\Phi_{\mathbb{R}}$ is a homeomorphism.

Remark 4.1. An evidence of this conjecture is given in [14], where a piecewise linear formula of the inverse map of $\Phi_{\mathbb{R}}$ is obtained when $r_{j}=1$ for any $j$. Recently, a piecewise linear formula of the shapes of rigged configurations in the same cases was conjectured as the tropicalization of ratios of so-called cylindric loop Schur functions and proven for the first shape in [15]. It is highly probable that such piecewise linear formulas exist in both ways for any tensor product of KR crystals, thereby proving the above conjecture. 
Remark 4.2. Although we introduced $\mathcal{P}_{\mathbb{R}}(\mathcal{B})$ by using the similarity map, it is also motivated from geometric crystals $[1,13,8]$, where the tropicalization of the corresponding geometric crystals produces a similar object.

Remark 4.3. A similar map to our $\Phi_{\mathbb{R}}$ in the case of $A_{1}$ has been constructed in [22] to linearize a certain integrable system called the tropical periodic Toda lattice. It would be interesting to establish an explicit connection between them.

Acknowledgements. The author thanks Atsuo Kuniba, Anne Schilling, Taichiro Takagi and Yasuhiko Yamada for useful discussion or comments. He also thanks the referees for careful reading of the manuscript and comments. He is partially supported by the Grants-in-Aid for Scientific Research No. 23340007 from JSPS.

\section{REFERENCES}

[1] A. Berenstein and D. Kazhdan, Geometric and unipotent crystals, GAFA 2000 (Tel Aviv, 1999), Geom. Funct. Anal. Special Volume, Part I, (2000) 188-236.

[2] G. Hatayama, A. Kuniba, M. Okado, T. Takagi and Z. Tsuboi, Paths, crystals and fermionic formulae, MathPhys Odyssey 2001, 205-272, Prog. Math. Phys. 23, Birkhäuser Boston, Boston, MA, 2002.

[3] G. Hatayama, A. Kuniba, M. Okado, T. Takagi and Y. Yamada, Remarks on fermionic formula, Contemporary Math. 248 (1999) 243-291.

[4] V. Kac, Infinite dimensional Lie algebras, 3rd ed., Cambridge University Press, 1990.

[5] S-J. Kang, M. Kashiwara, K. C. Misra, T. Miwa, T. Nakashima and A. Nakayashiki, Affine crystals and vertex models, Int. J. Mod. Phys. A 7 (suppl. 1A) (1992) 449-484.

[6] S.-J. Kang, M. Kashiwara, K. C. Misra, T. Miwa, T. Nakashima and A. Nakayashiki, Perfect crystals of quantum affine Lie algebras, Duke Math. J. 68 (1992) 499-607.

[7] M. Kashiwara and T. Nakashima, Crystal graphs for representations of the q-analogue of classical Lie algebras, J. Alg. 165 (1994) 295-345.

[8] M. Kashiwara, T. Nakashima and M. Okado, Affice geometric crystals and limit of perfect crystals, Trans. Amer. Math. Soc. 360 (2008) no.7, 3645-3686.

[9] S. V. Kerov, A. N. Kirillov and N. Yu. Reshetikhin, Combinatorics, the Bethe ansatz and representations of the symmetric group, Zap.Nauchn. Sem. (LOMI) 155 (1986) 50-64. (English translation: J. Sov. Math. 41 (1988) 916-924.)

[10] A. N. Kirillov and N. Yu. Reshetikhin, The Bethe ansatz and the combinatorics of Young tableaux, J. Sov. Math. 41 (1988) 925-955.

[11] A. N. Kirillov, A. Schilling and M. Shimozono, A bijection between Littlewood-Richardson tableaux and rigged configurations, Selecta Math. (N.S.) 8 (2002) no. 1, 67-135.

[12] A. Kuniba, M. Okado, R. Sakamoto, T. Takagi and Y. Yamada, Crystal interpretation of Kerov-Kirillov-Reshetikhin bijection, Nucl. Phys. B740 (2006) 299-327.

[13] A. Kuniba, M. Okado, T. Takagi and Y. Yamada, Geometric crystals and tropical $\mathcal{R}$ for $D_{n}^{(1)}$, IMRN, no.48, (2003) 2565-2620.

[14] A. Kuniba, R. Sakamoto and Y. Yamada, Tau functions in combinatorial Bethe ansatz, Nucl. Phys. B786 (2007) 207-266.

[15] T. Lam, P. Pylyavskyy and R. Sakamoto, Rigged configurations and cylindric loop Schur functions, arXiv:1410.4455.

[16] M. Okado, $X=M$ conjecture, MSJ Memoirs 17 (2007) 43-73.

[17] M. Okado, Simplicity and similarity of Kirillov-Reshetikhin crystals, Contemp. Math. 602 (2013) 183-194.

[18] M. Okado and A. Schilling, Existence of Kirillov-Reshetikhin crystals for nonexceptional types, Representation Theory 12 (2008) 186-207.

[19] M. Okado, A. Schilling and M. Shimozono, A tensor product theorem related to perfect crystals, J. Algebra 267 (2003) 212-245.

[20] A. Schilling, $X=M$ theorem: Fermionic formulas and rigged configurations under review, MSJ Memoirs 17 (2007) 75-104.

[21] M. Shimozono, Affine type A crystal structure on tensor products of rectangles, Demazure characters, and nilpotent varieties, J. Algebraic Combin. 15 (2002) no. 2, 151-187. 
[22] T. Takagi, Combinatorial aspects of the conserved quantities of the tropical periodic Toda lattice, J. Phys. A: Math. Theor. 47 (2014) 395201 (25pp).

[23] D. Takahashi and J. Satsuma, A soliton cellular automaton, J. Phys. Soc. Jpn. 59 (1990) $3514-3519$

Department of Mathematics, Osaka City University, 3-3-138, Sugimoto, Sumiyoshi-ku, OSAKA, 558-8585, JAPAN

E-mail address: okado@sci.osaka-cu.ac.jp 